left ventricular regional wall motion abnormalities (RWMA) can be reduced.

Ten patients prone to IDH were randomized to standard thrice-weekly hemodialysis with a dialysate temperature of either $37^{\circ} \mathrm{C}\left(\mathrm{HD}_{37}\right)$ or $35^{\circ} \mathrm{C}$ $\left(\mathrm{HD}_{35}\right)$. Patients were blinded to dialysate temperature. During the second week of the trial, patients underwent a dialysis session monitored with serial echocardiography and noninvasive hemodynamic monitoring. Patients crossed over to the other dialysis modality at the end of week 2 , and following a week on the new modality underwent a second monitored session. A questionnaire was used to score thermal symptoms.

Mean blood pressure was significantly lower with $\mathrm{HD}_{37}$ than with $\mathrm{HD}_{35}(P<0.001)$ and there were considerably more asymptomatic IDH events with the higher temperature dialysate owing to a lower peripheral resistance. Overall, 49 new RWMA occurred in 9 patients receiving $\mathrm{HD}_{37}$, while only 13 new RWMA occurred in 4 patients during $\mathrm{HD}_{35}$ (odds ratio $3.8,95 \% \mathrm{Cl}$ 2.1-6.9). Most patients tolerated $\mathrm{HD}_{35}$ well, with only three patients experiencing uncomfortable symptoms of cold. Further studies seem warranted to determine the dialysate temperature at which the benefits of IDH reduction are maximized and thermal symptoms minimized.

Original article Selby NM et al. (2006) Dialysis-induced regional left ventricular dysfunction is ameliorated by cooling the dialysate. Clin J Am Soc Nephrol 1: 696-705

\section{Study supports the use of kidneys and livers donated after cardiac death}

A shortage of kidneys and livers for transplantation has driven interest in the use of organs from non-heartbeating donors (i.e. those donated after cardiac death [DCD]); however, DCD organs are subjected to a longer period of ischemia than those donated after brain death (DBD) and have, therefore, been considered suboptimal. Now, a retrospective analysis of longterm outcomes provides support for the use of DCD organs.

From the United Network for Organ Sharing database, Doshi and Hunsicker identified 44,035 adults who had received a kidney, and 24,688 who had received a liver, from a cadaveric donor; $3.0 \%$ and $1.4 \%$, respectively, received an organ from a 'controlled' DCD donor. For patients who received kidney transplants, patient and graft survival after 5 years and incidence of rejection at 6 months were similar for DCD and DBD allografts. Delayed graft function occurred more frequently in the DCD group, but the average duration of hospitalization was only 1 day longer. On the basis of these results, the authors encourage the use of DCD kidneys for transplantation.

With regard to liver transplantation, DCD organ recipients had inferior rates of patient and graft survival at 3 years when compared with patients who received DBD organs from donors $<60$ years old, but comparable rates to those in patients who received DBD grafts from donors aged $\geq 60$ years. The authors conclude that DCD liver transplantation is an acceptable option, especially for candidates who are likely to die while awaiting a DBD organ from a donor aged less than 60 years.

Original article Doshi MD and Hunsicker LG (2007) Short- and long-term outcomes with the use of kidneys and livers donated after cardiac death. Am J Transplant 7: 122-129 